generation, STROOP, Autobiographical Memory Interview) were assessed at baseline, after six ECT and at the end of the ECT course.

Results: The study is in progress and preliminary results (mood, neuropsychological function, seizure indexes) will be presented.

Conclusions: All four forms of ECT appear effective, but preliminary results suggest some forms may be advantageous in terms of a lower rate of cognitive side-effects. There is evidence for the clinical use of bifrontal ECT. Ultrabrief unilateral ECT may hold great promise for the future.

07-02

\section{TMS treatment for depression: overview of efficacy and report on a sham-controlled trial of twice daily left prefrontal rTMS}

\author{
P Mitchell ${ }^{1,2}$, C Loo ${ }^{1,2}$, G Malhi ${ }^{2,4}$, T McFarquhar ${ }^{1,2}$, \\ P Sachdev ${ }^{1,3}$ \\ 'School of Psychiatry, University of New South Wales, Sydney, Australia; ${ }^{2 B}$ Back \\ Dog Institute, Prince of Wales Hospital, Sydney, New South Wales, Australia; \\ ${ }^{3}$ Neuropsychiatric Institute, Prince of Wales Hospital, Sydney, New South Wales, \\ Australia; and ${ }^{4}$ Academic Discipline of Psychological Medicine, \\ Northern Clinical School, University of Sydney, Sydney, Australia
}

Background: The majority of clinical trials have reported positive statistical results for repetitive transcranial magnetic stimulation (rTMS) (compared with a sham control) in treating depression, but the results of many studies were not clinically impressive. Recent studies have explored strategies to optimize the efficacy of rTMS. One such strategy is to increase the frequency of treatment sessions. The efficacy of twicedaily sessions of rTMS has not been previously examined in sham-controlled trials.

Methods: Thirty-eight subjects with DSM-IV major depressive episode were randomly assigned to receive active or sham rTMS for 2 weeks, with two treatment sessions per weekday. Treatment was given to the left prefrontal cortex at $10 \mathrm{~Hz}, 30$ trains of $5 \mathrm{~s}, 110 \%$ motor threshold. Subjects were allowed to receive up to 6 weeks of active daily rTMS in an open extension. Mood and cognitive functioning were assessed weekly during the study.

Results: The active treatment group improved more than the sham treatment group over the 2-week shamcontrolled period on Montgomery-Asberg Depression Rating Scale (MADRS) $(P<0.05)$ but not Hamilton Depression Rating scales. After 6 weeks of active treatment, $53 \%$ and $47 \%$ of subjects achieved response ( $\geq 50 \%$ improvement) and remission (MADRS $\leq 10$ ), respectively. rTMS was well tolerated.
Conclusion: High-frequency left prefrontal rTMS given twice a day was safe and more effective than sham in treating depression.

07-03

\section{Vagus nerve stimulation for treatment- resistant depression: utility and possible mechanisms of action}

\author{
JN Trollor ${ }^{1,2}$ \\ 'School of Psychiatry, University of New South Wales, Sydney, Australia; and \\ 2Prince of Wales Hospital, Sydney, New South Wales, Australia
}

Early clinical observations and subsequent prospective studies indicated that vagus nerve stimulation (VNS) had the potential to improve mood in patients with epilepsy. Subsequent studies have evaluated the effect of VNS in treatment-resistant major depression. These initial studies indicate a significant short- and long-term benefit of VNS on mood. Although the precise mechanisms underlying the antidepressant effect of VNS remain obscure, there is emerging evidence that VNS is associated with alteration of cerebrospinal fluid concentrations of various neurotransmitters. Furthermore, VNS impacts on functional activity of brain areas within the limbic system. This presentation will review the evidence for VNS as an antidepressant treatment and will review the potential neurobiological correlates of this effect. This will be compared with data from other brain stimulation approaches to treatment of depression. The implications of VNS for our understanding of functional models of depression will be discussed.

\section{7-04}

\section{Direct current and deep brain stimulation with lessons from neurosurgery}

\section{G Malhi' ${ }^{1,2,3}$, C Loo ${ }^{2,4}, \mathrm{~J} \mathrm{Lagopoulos}^{2,4}$, M Pigot $^{2,4}$, K Moss $^{2,4}$, P Mitchell ${ }^{2,4}$, P Sachdev ${ }^{4,5}$}

'Academic Discipline of Psychological Medicine, Northern Clinical School, University of Sydney; ${ }^{2}$ Black Dog Institute, Prince of Wales Hospital, Sydney, New South Wales, Australia; ${ }^{P}$ Prince of Wales Medical Research Institute, Sydney, New South Wales, Australia; ${ }^{4}$ School of Psychiatry, University of New South Wales, Sydney, Australia; and ${ }^{5}$ Neuropsychiatric Institute, Sydney, New South Wales, Australia

Neurosurgery for mental disorders (NMS) antedates pharmacotherapy and brain stimulation and arguably 